\title{
Editorial: Computational Modeling of Spintronic Materials
}

\author{
Xiaotian Wang ${ }^{1 *}$, Zhenxiang Cheng ${ }^{2}$ and Gokhan Surucu ${ }^{3,4 *}$ \\ ${ }^{1}$ School of Physical Science and Technology, Southwest University, Chongqing, China, ${ }^{2}$ Institute for Superconducting and \\ Electronic Materials (ISEM), University of Wollongong, Wollongong, NSW, Australia, ${ }^{3}$ Department of Physics, Middle East \\ Technical University, Ankara, Turkey, ${ }^{4}$ Department of Electric and Energy, Ahi Evran University, Kirsehir, Turkey
}

Keywords: first-principle calculations, density-functional theory, material simulation calculations, spintronic materials, electronic structrue

\section{Editorial on the Research Topic}

\section{Computational Modeling of Spintronic Materials}

In research topic "Computational Modeling of Spintronic Materials", we collected 17 articles dealing with themes as below:

i. Recent advances of spintronic materials;

ii. Frontiers in computational modeling of functional materials.

\section{OPEN ACCESS}

Edited by:

Roberto Brighenti,

University of Parma, Italy

Reviewed by:

Xiaojun Wu,

University of Science and Technology

of China, China

*Correspondence:

Xiaotian Wang

xiaotianwang@swu.edu.cn

Gokhan Surucu

g_surucu@yahoo.com

Specialty section:

This article was submitted to

Computational Materials Science,

a section of the journal

Frontiers in Materials

Received: 28 November 2020

Accepted: 14 December 2020

Published: 15 January 2021

Citation:

Wang X, Cheng Z and Surucu G (2021) Editorial: Computational Modeling of

Spintronic Materials.

Front. Mater. 7:634712.

doi: 10.3389/fmats.2020.634712
Heulser alloys are famous spintronic materials due to they usually host high Curie temperature and adjustable structures. Zhang et al. (Zhang et al., 2020) proposed quaternary Heusler compound $\mathrm{CoCrScSn}$ is a half-metal with $100 \%$ spin-polarization. Moreover, the elastic constants and halfmetallic states under different lattice constants are touched by Zhang et al. Classic Heusler ferromagnets consist of transition-group $\mathrm{d}$-metals and main-group p-elements. As a new direction of Heusler alloy, Wu et al. (Wu et al., 2020) studied a series of all-d-metal Heusler alloys $\mathrm{X}_{2} \mathrm{MnTi}(\mathrm{X}=\mathrm{Pd}, \mathrm{Pt}, \mathrm{Ag}, \mathrm{Au}, \mathrm{Cu}$ and $\mathrm{Ni})$. $\mathrm{X}_{2} \mathrm{MnTi}$ alloys are without main-group p-elements. Wu et al. also investigated the competition of the cubic $\mathrm{L} 2_{1}$ and tetragonal $\mathrm{L} 1_{0}$ states of these $\mathrm{X}_{2} \mathrm{MnTi}$ alloys. It is hoped that the possible martensitic transformation of these all-d-metal $\mathrm{X}_{2} \mathrm{MnTi}$ can be confirmed experimentally.

Half-metals have two spin-directions, one spin channel shows metallic property and the other one features insulating/semiconducting behaviors, resulting in 100\% spin-polarization. Also, half-metals can be used for efficient spin injection in spintronics. Doumi et al. (Doumi et al., 2020) proposed that $\mathrm{Ca}_{1-\mathrm{x}} \mathrm{Cr}_{\mathrm{x}} \mathrm{O}(x=0.25,0.5,0.75)$ are half-metals, where the ferromagnetism is mainly coming from the direct exchange splitting instead of the crystal field. Deng et al. (Deng et al., 2020) studied the magnetic structure and the Curie temperature of LiMgN with $\mathrm{Cu}$ doping, they found that these $\mathrm{Cu}$ doped LiMgN systems are dilute magnetic semiconductors with high Curie temperature. More interestingly, $\mathrm{Li}\left(\mathrm{Mg}_{0.875} \mathrm{Cu}_{0.125}\right) \mathrm{N}$ is predicted to be half-metal with a net magnetic moment. Chen et al. (Chen et al., 2020) investigated the electronic structures and magnetism of $\mathrm{Li}_{1 \pm y}\left(\mathrm{Mg}_{1-\mathrm{x}} \mathrm{Cr}_{\mathrm{x}}\right) \mathrm{P}$ $(x, y=0.125)$. They found that $\mathrm{Li}\left(\mathrm{Mg}_{0.875} \mathrm{Cr}_{0.125}\right) \mathrm{P}$ magnet is half-metal.

A series of materials co-exhibiting half-metallic state and topological elements are predicted via first-principles calculations. Chang et al. (Chang et al., 2020) proposed a rhombohedral type $\mathrm{GdMnO}_{3}$ is a half-metal with multiple Dirac-like band crossing points. Li (Li, 2020) proposed $\mathrm{CsCrCl}_{3}$ ferromagnet $\mathrm{P}_{3} / \mathrm{mmc}$ structure is a hypothetical half metal and it also belongs to nodal surface materials. Jia et al. (Jia et al., 2020) reported sandwich-like hexagonal $\mathrm{VI}_{3}$ monolayer is a halfmetal with Weyl fermions. Moreover, a series of topological semimetals and topological metals are reported in the research topic collection: 1) Zhang and Wang (Zhang and Wang, 2020) found that 
pure $\mathrm{Zr}$ is a topological material with type II nodal line and nodal surface states; 2) Li and Xia ( $\mathrm{Li}$ and Xia, 2020) reported that cubic $\mathrm{HfN}$ is a topological semimetal with zerodimensional (0-D) and one-dimensional (1-D) topological elements (TEs); 3) Li et al. (Li et al., 2020) proposed that tetragonal $\mathrm{PtO}$ is a topological material with nodal point and nodal line states; 4) $\mathrm{Xu}(\mathrm{Xu}, 2020)$ studied the electronic structures and the topological signatures of XPt $(X=\mathrm{Sc}, \mathrm{Y}$, and $\mathrm{La})$ via first-principles calculations. $\mathrm{XPt}(X=\mathrm{Sc}, \mathrm{Y}$ and La) materials are proposed to be novel systems with rich nodal line and nodal point states. Remarkably, we can observe opened and closed nodal lines, and triply degenerate and Dirac nodal points in these systems; 5) Xu et al. (Xu et al., 2020) predicted that hexagonal $\mathrm{Zr}_{3} \mathrm{X}(X=\mathrm{Al}, \mathrm{Ga}, \mathrm{In})$ are metallic systems with high stability and perfect $0-\mathrm{D}$ and 1-D TEs.

Some functional materials and their related physics behaviors are also investigated by first-principles calculations: 1) Ke et al. (Ke et al., 2020) performed a band-gap engineering work to study the band structures and band-gap tailoring of C-mono-doped, $\mathrm{C}-\mathrm{Ge}$, and C-Sn co-doped $\mathrm{GaN}$ nanosheets; 2) $\mathrm{Y}_{2} \mathrm{O}_{3}$ :Ce magnetic semiconductor with R-3 group symmetry was identified by Ju et al. (Ju et al., 2020) via CALYPSO, and the structural behaviors, doping site locations as well as electronic structures of $\mathrm{Y}_{2} \mathrm{O}_{3}: \mathrm{Ce}$ are also studied by $\mathrm{Ju}$ et al. via first-principles calculations in details; 3) Yan et al. (Yan et al., 2020) investigated the reaction pathways of $\mathrm{H}_{2}$ release from the hydrolytic $\mathrm{NH}_{3} \mathrm{BH}_{3}$ and examined the catalytic roles of small $\mathrm{NiCu}$ clusters; 4) Chen et al. (Chen et al., 2020) built an ultrathin van der Waals $\mathrm{Fe}_{3} \mathrm{GeTe}_{2} / \mathrm{In}_{2} \mathrm{Se}_{3}$ heterostructure and they reported that this heterostructure hosts tunable magnetic anisotropy and Dzyaloshinskii-Moriya Interaction (DMI).

We hope this research topic will attract readers. And we would like to thank all the authors, reviewers and editors who contributed to our research topic.

\section{AUTHOR CONTRIBUTIONS}

All authors listed have made a substantial, direct, and intellectual contribution to the work and approved it for publication.

\section{FUNDING}

This study has been funded by the National Natural Science Foundation of China (Grant No. 51801163), and the Fundamental Research Funds for the Central Universities (Grant No. XDJK 2019C112).

Conflict of Interest: The authors declare that the research was conducted in the absence of any commercial or financial relationships that could be construed as a potential conflict of interest.

Copyright (c) 2021 Wang, Cheng and Surucu. This is an open-access article distributed under the terms of the Creative Commons Attribution License (CC $B Y)$. The use, distribution or reproduction in other forums is permitted, provided the original author(s) and the copyright owner(s) are credited and that the original publication in this journal is cited, in accordance with accepted academic practice. No use, distribution or reproduction is permitted which does not comply with these terms. 